\title{
Cell multiplication: editorial overview
}

\author{
J.R. Pringle \\ Department of Biology, The University of Michigan, Ann Arbor, Michigan, USA \\ Current Opinion in Cell Biology 1989, 1:237-240
}

\section{A remarkable year for cell cycle studies}

Although the mechanisms and control of cellular reproduction have been studied for many years, it seems clear that the year under review has been one of the most remarkable yet in terms of the breadth and depth of the insights it has brought us. It also seems clear that this is no accident, and that we can expect more remarkable years of discovery in the near future. The reason is, of course, that the development of recombinant-DNA technology (in conjunction with other technical advances on a broad front) has triggered a revolution in cell biology that is comparable in its profundity to that brought on by the rapid development of cell fractionation and electron microscopy procedures in the late 1940s and early 1950s. The flames of the present revolution are being fanned by the growing appreciation of the degree to which fundamental mechanisms are conserved through. out the eukaryotic world.

The power of genetic and recombinant DNA approaches Although recombinant DNA technology has facilitated cell biological studics in many ways, its most profound impact derives from its having rendered practical each of two complementary genetic strategies, both of which are abundantly illustrated in the reviews that follow. One of these strategies, 'forward genetics', has of course been pursued vigorously throughout this century. It begins with the collection of interesting mutants, and has the great advantage that the mutations may affect proteins undreamt of by biochemists, too rare to be studied easily during waking hours, or both. Thus, totally unknown territories can be (and often have been) penetrated. However, until the advent of recombinant DNA technology, there was little real hope of charting these territories at the molecular level. Today, however, the mutational identification of an interesting gene can generally be followed by the physical isolation of that gene as a recombinant clone. This in turn leads to the sequence of the gene product, to its overproduction for facilitation of biochemical studies, and to the generation of antibodies that allow (among other things) the immunolocalization of the gene product. The information so obtained may not lead immediately to a molecular understanding of gene product function, but it certainly eases the path.
The altemative strategy, 'reverse genetics', has really only been thinkable since the advent of recombinant DNA technology. In this strategy, one begins with an interesting protein (or a messenger RNA with an interesting pattern of expression) and then uses an appropriate trick to isolate the corresponding gene. In vitro manipulations of the gene, followed by its transfection back into the appropriate cells, allow investigation of the functions in vivo of whole proteins and of their individual domains. The results are usually informative and sometimes startling, as in the discoveries that some eukaryotic cells can survive without clathrin (Payne and Schekman, Science 1985, 230:1009-1014; Lemmon and Jones, Science 1987, 238:504-509; Payne et al., J Cell Biol 1988, 106:1453-1461) and that Dictyostelium amoebae can crawl without myosin heavy chain (De Lozanne and Spudich, Science 1987, 236:1086-1091; Knecht and Loomis, Science 1987, 236:1081-1086).

\section{The conservation of fundamental mechanisms and its implications}

Although the past year has seen great progress on many fronts in the cell cycle field, surely the most remarkable discoveries relate to the control of mitosis and the realization of how closely the mechanisms of this control are conserved throughout the eukaryotic world. The Saccbaromyces cerevisiae CDC28 gene and the Scbizosaccharomyces pombe $\mathrm{CdC}^{+}$gene were originally identified by temperature-sensitive mutations that arrest cells at the critical $G_{1}$ control point known as Start (Hartwell et al., Science 1974, 183:46-51) and at mitosis (Nurse et al., Mol Gen Genet 1976, 146:167-178), respectively. Later work showed that $C D C 28$ was also involved in the control of mitosis (Piggott et al., Nature 1982, 298:391-393) and that $\mathrm{CdC2}^{+}$was also involved in the control of Start (Nurse and Bissett, Nature 1981, 292:558-560), and that the two genes were closely homologous and cross-functional (Beach et al., Nature 1982, 300:706-709; Booher and Beach, Mol Cell Biol 1986, 6:3523-3530), despite the vast phylogenetic gap between these species (Huysmans et al, Nucl Acids Res 1983, 11:2871-2880). This suggested that the fundamental mechanisms might be common to all eukaryotes, a view that was dramatically supported when Lee and Nurse (Nature 1987, 327:31-35) identified a human homologue by virtue of its ability to comple-

\footnotetext{
Abbreviations

CAMP - cyclic adenosine monophosphate; CAPK - CAMP dependent protein kinase; MPF - maturation-promoting factor.
} 
ment an $S$. pombe cdc2 mutation. This startling result was followed quickly by the equally startling discoveries that the Xenopus homologue of $C D C 28 / \mathrm{Cdc}^{+}$was a central component of the long-elusive maturation-promoting factor (MPF) and that the $C D C 28 / \mathrm{cdc}^{+} / \mathrm{MPF}$ homologues of various cell types all appear to interact with the local versions of 'cyclin', a protein originally detected by virtue of its striking pattern of continuous synthesis followed by abrupt post-mitotic breakdown in the cleaving eggs of marine invertebrates (see reviews by Fantes, pp 250-255 and Hunt, pp 268-274 in this issue).

These and other observations suggest that there is a deep conservation of fundamental mechanisms among eukary. otes; in some areas, this may extend to prokaryotes as well (see the reviews by Masters, pp 241-249 and Blow, pp 263-267). This in tum suggests that it is entirely appropriate to continue using a wide variety of cell types and experimental techniques to study fundamental cell biological problems. It's not so bad that Xenopus is a geneticist's nightmare if the proteins identified biochemically in frogs can be studied genetically in yeasts; it's not so bad that yeast chromosome cytology is difficult if the proteins identified genetically in yeast can be studied cytologically in mammalian cells. To pick just one concrete and topical example, the fascinating new proteins identified in mammalian cells by immunofluorescence screening of scleroderma and monoclonal antibodies (e.g. Cooke et al., J Cell Biol 1987, 105:2053-2057) will be difficult to understand if they can only be studied in mammalian cells. However, if homologues can be found in yeasts, then the exploration of protein function should be greatly facilitated.

In stressing the conservation of fundamental mechanisms, we should also not overlook the wide range of apparent variations in cell cycle organization and control as seen especially among the lower eukaryotes (see review by Berger, pp 256-262, in this issue). To the extent that these variations reflect only superficial differences in the expression of highly conserved mechanisms, they should nonetheless be instructive in revealing the degree of flexibility of those mechanisms. If deep differences in mechanism are involved, this will be all the more interesting, as there is clearly more to life than its least common denominator.

\section{The diversity of cell cycle problems and progress}

Studies of cellular reproduction must address two distinct categories of questions: (1) questions about the mechanics of the process (bow does a cell reproduce, once it has undertaken to do so?) and (2) questions about the overall control of the process (what determines whether and when a cell will reproduce?). In organising this series of reviews, we attempted to subdivide the cell cycle field in such a way that all major questions in both categories would be addressed. However, inevitably, this has not happened. There has been too much progress on too many different problems, and the constraints of time and space have forced the authors to be selective rather than comprehensive. Although their choices of topics to consider are all well justified, in many cases other choices could have been made with equal justification. In the remainder of this overview, I have attempted to fill in some of the gaps (with the explicit caveat that I have certainly not filled them all) and offered some of my own perceptions as to the status of certain problems in this field.

\section{The mechanics of cellular reproduction}

\section{Identification of cell cycle genes and proteins}

We cannot thoroughly understand any machine without a fairly complete inventory of its parts. The cell cycle is no exception, and a continuing effort to identify genes and gene products that function at specific stages of the cycle is clearly vital. In this regard, at least three developments are worthy of special note. First, recent successes suggest that it may soon be routine (even in cell types other than yeast!) to clone by complementation genes that have originally been identified by conditional-lethal mutations that arrest the cell cycle (Itrmann et al., Mol Cell Biol 1987, 7:3386-3393; Sekiguchi et al, EMBOJ 1988, 7:1683-1687; Osmani et al., Cell 1988, 53:237-244). Second, recent successes emphasize the potentially rich harvest obtainable by starting with conditional-lethal cell cycle mutations and seeking interacting genes using a battery of tactics including conventional suppression, suppression by overexpression of heterologous sequences, unlinked non-complementation, and synthetic lethality (Regan and Fuller, Gen Dev 1988, 2:82-92; Stearns and Botstein, Ge netics 1988, 119:249-260; Toda et al., Gen Dev 1988, 2:517-527; Adams et al., Science 1989, 243:231-233; Hays et al., Mol Cell Biol 1989, 9:875-884; Bender and Pringle, Proc Natl Acad Sci USA 1989, in press). Third, immunological screening using banks of scleroderma and monoclonal antibodies is beginning to identify many interesting proteins that can then be studied using reverse genetics (eg. Cooke et al., 1987; Snyder and Davis, Cell 1988, 54:743-754).

\section{Macromolecule synthesis through the cell cycle}

Heintz (pp 275-279) has reviewed the considerable progress made toward understanding the temporal regulation of gene expression during the mammalian cell cycle. It is worth noting that this issue is also being studied successfully in yeast and other lower eukaryotes (Breeden, Trends Genet 1988, 4:249-253; Berger, pp 256-262). However, it is also worth noting that the significance of such regulation in cell cycle progression and control remains problematic. In particular, both of two com. mon assumptions (that genes important in cell cycle control must exhibit temporal regulation of transcription; that genes exhibiting temporal regulation of transcription must be important in cell cycle control) are almost certainly wrong. Firstly, it seems abundantly clear that the levels of gene products can vary during the cell cycle for many reasons other than periodic transcription (Heintz, pp 275-278; Evans et al., Cell 1983, 33:389-396). Secondly, it seems equally clear that many proteins of indisputable importance in the regulation of cell cycle pro- 
gression vary little or not at all in their levels through the cycle (Aves et al., EMBO J 1985, 4:457-463; Simanis and Nurse, Cell 1986, 45:261-268; Brizuela et al, EMBOJ 1987, 6:3507-3514; Lee et al, Nature 1988, 333:676-679), are normally present (or can easily be tolerated) in vast excess over the amount needed to complete the cell cycle in progress (Byers and Sowder, J Cell Biol 1980, 87:6a; Sclafani et al. Mol Cell Biol 1988, 8:293-300), or both. Third, cells seem remarkably ambivalent about whether particular proteins are produced continuously or in a cell cycle-specific fashion (Creanor et al., J Cell Sci 1983, 61:339-349; White et al., EMBO J 1986, 5:1705-1709; Berger, pp 256-262) and remarkably tolerant of the continuous production of proteins that are normally produced in a cell cycle-specific fashion (Han et al., Cell 1987, 48:589-597; Han et ah, EMBO J 1988, 7:2211-2219; McIntosh et al., Mol Cell Biol 1988, 8:4616-4624). In summary, it appears that cells exploit mechanisms at all possible levels to regulate cell cycle progression, but that the most generally important mechanisms involve the regulation of gene product activity or assembly. Although such regulation no doubt involves numerous specific mechanisms, covalent modifications such as phosphorylation (Fantes, pp 250-255; Hunt, pp 268-274; Russell and Nurse, Cell 1987, 49:569-576) and ubiquitination (Murti et al., Proc Natl Acad Sci USA 1988, 85:3019-3023; Goebl et al., Science 1988, 241:1331-1335) seem certain to be important.

\section{Spatial organization in the cell cycle}

Interest in the temporal organization of the cell cycle should not blind us to the fact that the maintenance and generation of appropriate spatial organization during the cycle is equally important. Important progress in understanding morphogenesis in the cell cycle has been made both in bacteria (Sommer and Newton, $J$ Bacteriol 1988, 170:409-415; de Boer et al., J Bacteriol 1988, 170:2106-2112; Hahnenberger and Shapiro, $J$ Bacteriol 1988, 170:4119-4124) and in yeasts. In S. cerevisiae, we have learned much about the functions of the cytoplasmic microtubules, which are not involved in polar growth of the cell surface but do function in nuclear migration and spindle orientation (Huffaker et al., $J$ Cell Biol 1988, 106:1997-2010; Jacobs et al., J Cell Biol 1988, 107:1409-1426), as well as much about the proteins that associate with actin to carry out polarized cell surface growth (and perhaps other processes; Drubin et ah, J Cell Biol 1988, 107:2551-2561; Magdolen et al., Mol Cell Biol 1988, 8:5108-5115; Adams et al., 1989; Liu and Bretscher, Proc Natl Acad Sci USA 1989, 86:90-93). It is particularly pleasing that yeast appears to contain a full complement of the actin-associated proteins that have been described in other cell types, which should open this whole complicated system to facile genetic investigation. We have also begun to learn more about an apparently novel type of cytoskeletal element that functions in morphogenesis at the bud neck in $S$. cerevisiae but is probably present in other eukaryotic cells as well (Haarer and Pringle, $\mathrm{Mol}$ Cell Biol 1987, 7:3678-3687; Kim et al, J Cell Biol 1989, in press; Haarer et al., personal communication).

\section{Cell division}

Spindle function during mitosis was reviewed recently by Mitchison in this series (Curr Opin Cell Biol 1989, 1:67-74) and eukaryotic cytokinesis will by reviewed by Salmon in the next issue (Curr Opin Cell Biol 1989, in press). In addition, it should be noted that continuing progress is being made in understanding both prokaryotic cell division (de Boer et al, 1988; Nathan and Newton, $J$ Bacteriol 1988, 170:2319-2327; Phoenix and Drapeau, $J$ Bacteriol 1988, 170:4338-4342; Beall et al, J Bacteriol 1988, 170:4855-4864) and the mechanisms of disassembly and reassembly of the nuclear envelope during mitosis (Newport and Spann, Cell 1987, 48:219-230; Sheehan et al., $J$ Cell Biol 1988, 106:1-12; Wilson and Newport, J Cell Biol 1988, 107:57-68).

\section{The control of cellular reproduction}

\section{Growth control versus cell cycle control}

In considering the overall control of cellular reproduction, it is essential to remember the differences in lifestyle between unicellular organisms and the individual cells of multicellular organisms. The former are typically programmed to grow (i.e. increase in mass) as rapidly as the supply of energy and nutrients from the environment allows and to divide as often as the cell mass and essential components can be duplicated. In contrast, the latter must reproduce at rates consistent with their roles in the organism as a whole. Moreover, it is not only cell division but also cell growth that must be controlled - an uncontrolled increase in cell mass would be just as inappropriate in such cells as would uncontrolled division. This refusal to grow must be maintained even though the cells are (usually) bathed in a nutrient-replete environment in the organism. Thus, the cell's decision as to whether or not to reproduce is intimately tied to its decision as to whether or not to grow, and much interest attaches to the study of the 'growth factors' that seem to control both decisions (Westermark and Heldin, pp 279-285), as evidenced in part by the uncontrolled growth and division produced by oncogenes that resemble growth factors or their receptors (Sigal and Gibbs, pp 286-290).

The foregoing arguments suggest that unicellular organisms do not require growth control but only cell cy cle control. How then do we explain the function of the $S$. cerevisiae RAS/cyclic adenosine monophosphate (CAMP)/CAMP-dependent protein kinase (CAPK) system, mutational perturbations of which can clearly produce a $G_{1}$-specific block of the cell cycle, apparently by first blocking growth? The details of this complicated system of interacting components continue to be worked out with impressive speed (Toda et al, 1988; Marshall et al., Mol Cell Biol 1988, 8:52-61; Wilson and Tatchell, Mol Cell Biol 1988, 8:505-510; Powers et al, Mol Cell Biol 1989, 9:390-395). Two recent developments are particularly intriguing. First, cells appear to be reasonably normal if they totally lack CAMP, so long as they contain an unregulated but appropriately attenuated CAPK catalytic subunit (Cameron et al., Cell 1988, 53:555-566); this suggests 
the existence of CAMP-independent mechąnisms whose regulatory roles parallel those of the cAMP-dependent ones. Second, there is increasing evidence that $R A S$ plays a second role in yeast in addition to its role in regulating adenylate cyclase (Toda et al, Cell 1987, 50:277-287; Wigler et al, Cold Spring Harb Symp Quant Biol 1988, in press); this resurrects the possibility that direct information about the functions of ras in mammalian cells may be gained by studying yeast.

Despite this progress, the ultimate role of the $R A S / \mathrm{CAMP}$ /CAPK system in yeast physiology remains somewhat obscure. I think that the most reasonable view at present is that this system exists not for the overall control of growth but for coordination of its different aspects. That is, by allowing a coordinate shut-down (or activation) of different aspects of macromolecule synthesis, it allows the cell to avoid the unbalanced growth that might otherwise result as environmental conditions change.

\section{$\mathbf{G}_{1}$ control, mitotic control, and cell cycle oscillators}

The observation that certain subsets of cell cycle events can continue periodically even when other subsets of events are blocked has made the concept of a 'fundamental cell cycle oscillator' seductive (Creanor and Mitchison, J Cell Sci 1986, 86:207-215; Kimelman et al., Cell 1987, $48: 399-407)$. However, I think that this is a somewhat misleading perception based on: (1) insufficient attention to the degree to which the events of the cell cycle are organized as multiple interconnected chains of dependent events (Pringle and Hartwell, In The Molecular Biology of the Yeast Saccharomyces, edited by Strathern, Jones and Broach. Cold Spring Harbor Laboratory, 1981, pp 97-142; Hirano et al, EMBO J 1986, 5:2973-2979; Ohkura et al., $E M B O J$ 1988, 7:1465-1473), and (2) excessive attention to certain cell types that are highly programuned for rapid divisions. It is important to remember that the cleaving egg represents but a moment in the life cycle and that the nutrient-replete environments that we provide for microorganisms in the laboratory are presumably the exception rather than the rule in nature. For most cells, most of the time, each trip around the cell cycle is an adventure to be undertaken only when the environment has been carefully assessed for suitability. A substantial weight of evidence suggests that this assessment normally occurs at a control point in $\mathrm{G}_{1}$.

This line of reasoning thus also leads me to a viewpoint that may seem rather heterodox in the current flush of (entirely justifiable) excitement about mitotic control (see above). That is, I think it is reasonable to regard this control more as a checkpoint (or coordination point) in a system that is already committed to reproduction than as a point at which fundamental decisions about whether to reproduce or not are taken. After all, even in $S$. pombe, where mitotic control has been so elegantly studied (Fantes, pp 250-255), nutritional limitation (Nasmyth, $J$ Cell Sci 1979, 36:155-168) or the need to choose between asexual and sexual reproduction (Nurse and Bissett, 1981) quickly makes manifest the existence of a decision point in $G_{1}$. It is only fair to note that this argument begs the fascinating questions of how and why the same proteins (the $S$. cerevisiae $C D C 28$ and $S$. pombe $\mathrm{CdC2}^{+}$ products) function both in mitotic control and at Start in $G_{1}$.

\section{$G_{0}$ versus $G_{1}$}

It has long seemed reasonable to suppose that cells that opt not to reproduce exit from the cell cycle (normally from $G_{1}$ ) into a discrete $G_{0}$ state. Although non-proliferating cells do indeed generally differ from actively proliferating cells in many ways, it has proven surprisingly difficult to identify unequivocally gene products that function specifically in the transition to or from the hypothetical $G_{0}$ state. For example, after a long search, Johnston, Singer and colleagues finally identified a yeast mu tant that seemed to be defective specifically in the $G_{0}$ to $\mathrm{G}_{1}$ transition (Drebot et al., Proc Natl Acad Sci USA 1987, $84 ; 7948-7952)$. However, genetic analysis of this mutant revealed that it contained two unlinked mutations, one of which blocked the cell cycle of actively proliferating cells as well as the first cycle upon emergence from stationary phase, and the second of which suppressed the first in actively proliferating cells. Thus, it seems unlikely that either of the genes identified is specifically involved in the $G_{0}$ to $G_{1}$ transition.

An alternative approach is to screen for proteins or messenger RNAs that are expressed differentially in nonproliferating or proliferating cells (or during transitions between the two states), and indeed many potentially interesting species have been found in this way (Lau and Nathans, Proc Natl Acad Sci USA 1987, 84:1182-1186; Philipova et al., Biol Cell 1987, 60:1-8; Tominaga, FEBS Lett 1987, 226:53-57; Wang, J Cell Pby siol 1987, 133:151-157; Ching and Wang, Proc Natl Acad Sci USA 1988, 85:151-155; Ryder et al., Proc Natl Acad Sci USA 1988, 85:1487-1491; Rollins et al., Proc Natl Acad Sci USA 1988, 85:3738-3742; Schneider et al., Cell 1988, 54:787-793; Linzer and Mordacq, EMBO J 1988, 6:2281-2288; Ryseck et al., Exp Cell Res 1989, 180:266-275; Ryseck et al., Exp Cell Res 1989, 180:537-545; and references cited therein). However, it seems fair to say that it remains unclear (and may be rather hard to determine) how many of these species, if any, actually function in regulating the transitions between the proliferating and non-proliferating states. The application of reverse genetic procedures to sporulating yeast has shown that inferring differential function from differential expression is a risky business (Kaback and Feldberg, Mol Cell Biol 1985, 5:751-761; Yamashita and Fukui, Mol Cell Biol 1985, 5:3069-3073; Law and Segall, Mol Cell Biol 1988, 8:912-922).

\section{Conclusions}

The pace of discovery in the cell cycle field during the past year has been staggering. However, as should be apparent from this and the reviews that follow, there is also no dearth of interesting problems left to study. This field will clearly keep us busy for many years to come. 